\title{
STABILITY ON THE ONE-DIMENSIONAL INVERSE SOURCE SCATTERING PROBLEM IN A TWO-LAYERED MEDIUM
}

\author{
YUE ZHAO AND PEIJUN LI
}

\begin{abstract}
This paper concerns the stability on the inverse source scattering problem for the onedimensional Helmholtz equation in a two-layered medium. We show that the increasing stability can be achieved by using multi-frequency wave field at the two end points of the interval which contains the compact support of the source function.
\end{abstract}

\section{INTRODUCTION AND PROBLEM FORMULATION}

We consider the one-dimensional Helmholtz equation in a two-layered medium:

$$
u^{\prime \prime}(x, \omega)+\kappa^{2}(x) u(x, \omega)=f(x), \quad x \in(-1,1),
$$

where $\omega>0$ is the angular frequency, the source function $f$ has a compact support which is assumed to be contained in the interval $(-1,1)$, and the wave number $\kappa$ satisfies

$$
\kappa(x)= \begin{cases}\kappa_{1}, & x>0 \\ \kappa_{2}, & x<0\end{cases}
$$

Here $\kappa_{j}=c_{j} \omega, j=1,2$, where $c_{j}>0$ are constants. The wave field $u$ is required to satisfy the outgoing wave conditions:

$$
u^{\prime}(-1, \omega)+\mathrm{i} \kappa_{2} u(-1, \omega)=0, \quad u^{\prime}(1, \omega)-\mathrm{i} \kappa_{1} u(1, \omega)=0 .
$$

Given $f \in L^{2}(-1,1)$, it is known that the problem (1.1)-(1.2) has a unique solution:

$$
u(x, \omega)=\int_{0}^{1} g(x, y) f(y) \mathrm{d} y,
$$

where $g$ is the Green function given as follows

$$
g(x, y)=\left\{\begin{array}{ll}
\mathrm{i} \frac{\kappa_{1}-\kappa_{2}}{2 \kappa_{1}\left(\kappa_{1}+\kappa_{2}\right)} e^{\mathrm{i} \kappa_{1}(x+y)}+\frac{\mathrm{i}}{2 \kappa_{1}} e^{\mathrm{i} \kappa_{1}|x-y|}, & x>0, \\
\frac{\mathrm{i}}{\kappa_{1}+\kappa_{2}} e^{\mathrm{i}\left(\kappa_{1} y-\kappa_{2} x\right)}, & x<0,
\end{array} \text { for } y>0\right.
$$

and

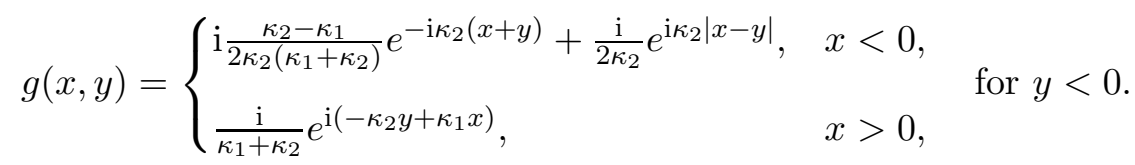

This paper concerns the inverse source problem: Let $f$ be a complex function with a compact support contained in $(-1,1)$. The inverse problem is to determine $f$ by using the boundary data $u(-1, \omega)$ and $u(1, \omega)$ with $\omega \in(0, K)$ where $K>1$ is a positive constant.

The inverse source scattering problem have significant applications in antenna synthesis, medical imaging, and optical tomography [4,13. They been extensively investigated by many researchers [1] 3, 5, 9, 11. It is known that there is no uniqueness for the inverse source problems at a fixed frequency due to the existence of non-radiating sources [10,12. Recently, it has been realized that the use of multi-frequency data can not only overcome the difficulties of non-uniqueness, which

2010 Mathematics Subject Classification. 78A46, 35R30.

Key words and phrases. inverse source problem, the Helmholtz equation, two-layered medium, stability.

The research of PL was supported in part by the NSF grant DMS-1151308. 
are presented at a single frequency, but also achieve increasing stability [6, 8, 14, 15]. These work assume that the medium is homogeneous in the whole space. In this work, we intend to establish the increasing stability on the inverse source problem for the one-dimensional Helmholtz equation in a two-layered medium.

\section{MAIN RESUlT}

Define a functional space:

$$
\mathcal{F}_{M}=\left\{f \in H^{n}(-1,1):\|f\|_{H^{n}(-1,1)} \leq M, \operatorname{supp} f \subset(-1,1)\right\},
$$

where $n \in \mathbb{N}$ and $M>1$ is a constant. Hereafter, the notation " $a \lesssim b$ " stands for $a \leq C b$, where $C$ is a generic constant independent of $n, \omega, K, M$, but may change step by step in the proofs.

The following stability estimate is the main result of this paper.

Theorem 2.1. Let $f \in \mathcal{F}_{M}$ and let $u$ be the solution (1.3) corresponding to $f$. Then we have

$$
\|f\|_{L^{2}(-1,1)}^{2} \lesssim \epsilon^{2}+\frac{M^{2}}{\left(\frac{K^{\frac{2}{3}}|\ln \epsilon|^{\frac{1}{4}}}{(6 n-3)^{3}}\right)^{2 n-1}},
$$

where

$$
\epsilon=\left(\int_{0}^{K} \omega^{2}\left(|u(-1, \omega)|^{2}+|u(1, \omega)|^{2}\right) \mathrm{d} \omega\right)^{\frac{1}{2}} .
$$

Remark 2.2. The stability estimate (2.1) consists of two parts: the data discrepancy and the high frequency tail. The former is of the Lipschitz type. The latter decreases as K increases which makes the problem have an almost Lipschitz stability. The result explains that the problem becomes more stable when higher frequency data is used. The stability estimate (2.1) also implies the uniqueness of the inverse source problem.

\section{Proof of Theorem 2.1}

Consider the following two functions:

$$
f_{1}(x)=\left\{\begin{array}{ll}
f(x), & x>0, \\
0, & x<0,
\end{array} \text { and } f_{2}(x)= \begin{cases}0, & x>0 \\
f(x), & x<0 .\end{cases}\right.
$$

Lemma 3.1. Let $f \in L^{2}(-1,1)$ with $\operatorname{supp} f \subset(-1,1)$. We have

$$
\|f\|_{L^{2}(-1,1)}^{2} \lesssim \int_{0}^{+\infty} \omega^{2}\left(|u(-1, \omega)|^{2}+|u(1, \omega)|^{2}\right) \mathrm{d} \omega .
$$

Proof. Choosing $\xi_{1} \in \mathbb{R}$ with $\left|\xi_{1}\right|=\kappa_{1}$, multiplying both sides of (1.1) by $e^{-\mathrm{i} \xi_{1} x}$, and integrating over $(0,1)$ with respect to $x$, we have from the integration by parts that

$$
e^{-\mathrm{i} \xi_{1}} u^{\prime}(1, \omega)+\mathrm{i} \xi_{1} e^{-\mathrm{i} \xi_{1}} u(1, \omega)-u^{\prime}(0, \omega)-\mathrm{i} \xi_{1} u(0, \omega)=\int_{0}^{1} e^{-\mathrm{i} \xi_{1} x} f_{1}(x) \mathrm{d} x .
$$

Similarly, choosing $\xi_{2} \in \mathbb{R}$ with $\left|\xi_{2}\right|=\kappa_{2}$, multiplying both sides of (1.1) by $e^{-\mathrm{i} \xi_{2} x}$, and integrating over $(-1,0)$ with respect to $x$, we have from the integration by parts that

$$
-e^{-\mathrm{i} \xi_{2}} u^{\prime}(-1, \omega)-\mathrm{i} \xi_{2} e^{\mathrm{i} \xi_{2}} u(-1, \omega)+u^{\prime}(0, \omega)+\mathrm{i} \xi_{2} u(0, \omega)=\int_{-1}^{0} e^{-\mathrm{i} \xi_{2} x} f_{2}(x) \mathrm{d} x .
$$

It follows from (1.3) and (3.1) that

$$
u(x, \omega)=\int_{0}^{1} g(x, y) f(y) \mathrm{d} y=\int_{0}^{1} g(x, y) f_{1}(y) \mathrm{d} y+\int_{-1}^{0} g(x, y) f_{2}(y) \mathrm{d} y,
$$


which gives

$$
\begin{aligned}
u(0, \omega) & =\int_{0}^{1} g(0, y) f_{1}(y) \mathrm{d} y+\int_{-1}^{0} g(0, y) f_{2}(y) \mathrm{d} y \\
& =\int_{0}^{1} \frac{\mathrm{i}}{\kappa_{1}+\kappa_{2}} e^{\mathrm{i} \kappa_{1} y} f_{1}(y) \mathrm{d} y+\int_{-1}^{0} \frac{\mathrm{i}}{\kappa_{1}+\kappa_{2}} e^{-\mathrm{i} \kappa_{2} y} f_{2}(y) \mathrm{d} y .
\end{aligned}
$$

On the other hand, we have from a simple calculation that

$$
\begin{aligned}
u^{\prime}(0, \omega) & =\int_{0}^{1} g^{\prime}(0, y) f_{1}(y) \mathrm{d} y+\int_{-1}^{0} g^{\prime}(0, y) f_{2}(y) \mathrm{d} y \\
& =\int_{0}^{1} \frac{\kappa_{2}}{\kappa_{1}+\kappa_{2}} e^{\mathrm{i} \kappa_{1} y} f_{1}(y) \mathrm{d} y+\int_{-1}^{0} \frac{-\kappa_{1}}{\kappa_{1}+\kappa_{2}} e^{-\mathrm{i} \kappa_{2} y} f_{2}(y) \mathrm{d} y .
\end{aligned}
$$

Letting $\xi_{1}=-\kappa_{1}$, we have from (3.4) and (3.5) that

$$
u^{\prime}(0, \omega)-\mathrm{i} \kappa_{1} u(0, \omega)=\int_{0}^{1} e^{\mathrm{i} \kappa_{1} y} f_{1}(y) \mathrm{d} y
$$

Combining (3.6) and (3.2), we obtain

$$
e^{\mathrm{i} \kappa_{1}} u^{\prime}(1, \omega)-\mathrm{i} \kappa_{1} e^{\mathrm{i} \kappa_{1}} u(1, \omega)=2 \int_{0}^{1} e^{\mathrm{i} \kappa_{1} x} f_{1}(x) \mathrm{d} x,
$$

Using the outgoing radiation condition (1.2), we get from the above equation that

$$
e^{\mathrm{i} \kappa_{1}} \mathrm{i} \kappa_{1} u(1, \omega)-\mathrm{i} \kappa_{1} e^{\mathrm{i} \kappa_{1}} u(1, \omega)=2 \int_{0}^{1} e^{\mathrm{i} \kappa_{1} x} f_{1}(x) \mathrm{d} x,
$$

which implies

$$
\left|\hat{f}_{1}\left(-\kappa_{1}\right)\right|^{2} \lesssim \omega^{2}|u(\omega, 1)|^{2}
$$

Letting $\xi_{2}=\kappa_{2}$, we have from (3.4) and (3.5) that

$$
u^{\prime}(0, \omega)+\mathrm{i} \kappa_{2} u(0, \omega)=-\int_{-1}^{0} e^{-\mathrm{i} \kappa_{2} y} f_{2}(y) \mathrm{d} y,
$$

Combining (3.8), (3.3), and (1.2), we obtain

$$
e^{-\mathrm{i} \kappa_{2}} \mathrm{i} \kappa_{2} u^{\prime}(-1, \omega)-\mathrm{i} \kappa_{2} e^{\mathrm{i} \kappa_{2}} u(-1, \omega)=2 \int_{-1}^{0} e^{-\mathrm{i} \kappa_{2} x} f_{2}(x) \mathrm{d} x
$$

which shows

$$
\left|\hat{f}_{2}\left(\kappa_{2}\right)\right|^{2} \lesssim \omega^{2}|u(\omega,-1)|^{2} .
$$

Letting $\xi_{1}=\kappa_{1}$, we get from (3.4) and (3.5) that

$$
\begin{aligned}
u^{\prime}(0, \omega)+\mathrm{i} \kappa_{1} u(0, \omega) & =\int_{0}^{1} \frac{\kappa_{2}-\kappa_{1}}{\kappa_{1}+\kappa_{2}} e^{\mathrm{i} \kappa_{1} y} f_{1}(y) \mathrm{d} y-\int_{-1}^{0} \frac{2 \kappa_{1}}{\kappa_{1}+\kappa_{2}} e^{-\mathrm{i} \kappa_{2} y} f_{2}(y) \mathrm{d} y \\
& =\frac{\kappa_{2}-\kappa_{1}}{\kappa_{1}+\kappa_{2}} \hat{f}_{1}\left(-\kappa_{1}\right)-\frac{2 \kappa_{1}}{\kappa_{1}+\kappa_{2}} \hat{f}_{2}\left(\kappa_{2}\right) .
\end{aligned}
$$

It follows from (3.10), (3.2), and (1.2) that we obtain

$$
e^{-\mathrm{i} \kappa_{1}} \mathrm{i} \kappa_{1} u(1, \omega)+\mathrm{i} \kappa_{1} e^{-\mathrm{i} \kappa_{1}} u(1, \omega)-\frac{\kappa_{2}-\kappa_{1}}{\kappa_{1}+\kappa_{2}} \hat{f}_{1}\left(-\kappa_{1}\right)+\frac{2 \kappa_{1}}{\kappa_{1}+\kappa_{2}} \hat{f}_{2}\left(\kappa_{2}\right)=\hat{f}_{1}\left(\kappa_{1}\right),
$$

which means

$$
\left|\hat{f}_{1}\left(\kappa_{1}\right)\right|^{2} \lesssim \omega^{2}|u(1, \omega)|+\left|\hat{f}_{1}\left(-\kappa_{1}\right)\right|^{2}+\left|\hat{f}_{2}\left(\kappa_{2}\right)\right|^{2}
$$


Finally, letting $\xi_{2}=-\kappa_{2}$, we have from (3.4) and (3.5) that

$$
\begin{aligned}
u^{\prime}(0, \omega)-\mathrm{i} \kappa_{2} u(0, \omega) & =\int_{0}^{1} \frac{2 \kappa_{2}}{\kappa_{1}+\kappa_{2}} e^{\mathrm{i} \kappa_{1} y} f_{1}(y) \mathrm{d} y+\int_{-1}^{0} \frac{\kappa_{2}-\kappa_{1}}{\kappa_{1}+\kappa_{2}} e^{-\mathrm{i} \kappa_{2} y} f_{2}(y) \mathrm{d} y \\
& =\frac{2 \kappa_{2}}{\kappa_{1}+\kappa_{2}} \hat{f}_{1}\left(-\kappa_{1}\right)+\frac{\kappa_{2}-\kappa_{1}}{\kappa_{1}+\kappa_{2}} \hat{f}_{2}\left(\kappa_{2}\right) .
\end{aligned}
$$

Using (3.12), (3.3), and (1.2), we have

$$
e^{\mathrm{i} \kappa_{2}} \mathrm{i} \kappa_{2} u(-1, \omega)+\mathrm{i} \kappa_{2} e^{-\mathrm{i} \kappa_{2}} u(-1, \omega)+\frac{2 \kappa_{2}}{\kappa_{1}+\kappa_{2}} \hat{f}_{1}\left(-\kappa_{1}\right)+\frac{\kappa_{2}-\kappa_{1}}{\kappa_{1}+\kappa_{2}} \hat{f}_{2}\left(\kappa_{2}\right)=\hat{f}_{2}\left(-\kappa_{2}\right),
$$

which means

$$
\left|\hat{f}_{2}\left(-\kappa_{2}\right)\right|^{2} \lesssim \omega^{2}|u(-1, \omega)|^{2}+\left|\hat{f}_{1}\left(-\kappa_{1}\right)\right|+\left|\hat{f}_{2}\left(\kappa_{2}\right)\right| .
$$

Therefore, it follows from (3.7) that we get

$$
\int_{0}^{\infty}\left|\hat{f}_{1}\left(-\kappa_{1}\right)\right|^{2} \mathrm{~d} \omega \lesssim \int_{0}^{\infty} \omega^{2}|u(\omega, 1)|^{2} \mathrm{~d} \omega
$$

Using (3.9) gives

$$
\int_{0}^{\infty}\left|\hat{f}_{2}\left(\kappa_{2}\right)\right|^{2} \mathrm{~d} \omega \lesssim \int_{0}^{\infty} \omega^{2}|u(-1, \omega)|^{2} \mathrm{~d} \omega
$$

It follows from (3.11), (3.14), and (3.15) that we have

$$
\int_{0}^{\infty}\left|\hat{f}_{1}\left(\kappa_{1}\right)\right|^{2} \mathrm{~d} \omega \lesssim \int_{0}^{\infty} \omega^{2}|u(-1, \omega)|^{2} \mathrm{~d} \omega+\int_{0}^{\infty} \omega^{2}|u(1, \omega)|^{2} \mathrm{~d} \omega ;
$$

Finally following from (3.13), (3.14), and (3.15), we obtain

$$
\int_{0}^{\infty}\left|\hat{f}_{2}\left(-\kappa_{2}\right)\right|^{2} \mathrm{~d} \omega \lesssim \int_{0}^{\infty} \omega^{2}|u(-1, \omega)|^{2} \mathrm{~d} \omega+\int_{0}^{\infty} \omega^{2}|u(1, \omega)|^{2} \mathrm{~d} \omega .
$$

We obtain from the Plancherel theorem that

$$
\|f\|_{L^{2}(-1,1)}^{2}=\|f\|_{L^{2}(-\infty, \infty)}^{2}=\|\hat{f}\|_{L^{2}(-\infty, \infty)}^{2} \lesssim\left\|\hat{f}_{1}\right\|_{L^{2}(-\infty, \infty)}^{2}+\left\|\hat{f}_{2}\right\|_{L^{2}(-\infty, \infty)}^{2} .
$$

On the other hand, we have

$$
\left\|\hat{f}_{1}\right\|_{L^{2}(-\infty, \infty)}^{2}=\int_{0}^{\infty}\left|\hat{f}_{1}(\omega)\right|^{2} \mathrm{~d} \omega+\int_{0}^{\infty}\left|\hat{f}_{1}(-\omega)\right|^{2} \mathrm{~d} \omega .
$$

Using (3.14) and (3.16) yields

$$
\left\|\hat{f}_{1}\right\|_{L^{2}(-\infty, \infty)}^{2} \lesssim \int_{0}^{+\infty} \omega^{2}\left(|u(-1, \omega)|^{2}+|u(1, \omega)|^{2}\right) \mathrm{d} \omega .
$$

Similarly, we have from (3.15) and (3.17) that

$$
\left\|\hat{f}_{2}\right\|_{L^{2}(-\infty, \infty)}^{2} \lesssim \int_{0}^{+\infty} \omega^{2}\left(|u(-1, \omega)|^{2}+|u(1, \omega)|^{2}\right) \mathrm{d} \omega .
$$

The proof is completed by combining the above estimates.

Lemma 3.2. Let $f \in L^{2}(-1,1)$. We have

$$
\begin{aligned}
\omega^{2}|u(-1, \omega)|^{2} & \lesssim\left|\int_{0}^{1} e^{\mathrm{i} c_{1} \omega y} f_{1}(y) \mathrm{d} y\right|^{2}+\left|\int_{-1}^{0} e^{-\mathrm{i} c_{2} \omega y} f_{2}(y) \mathrm{d} y\right|^{2}+\left|\int_{-1}^{0} e^{\mathrm{i} c_{2} \omega y} f_{2}(y) \mathrm{d} y\right|^{2}, \\
\omega^{2}|u(1, \omega)|^{2} & \lesssim\left|\int_{0}^{1} e^{\mathrm{i} c_{1} \omega y} f_{1}(y) \mathrm{d} y\right|^{2}+\left|\int_{0}^{1} e^{-\mathrm{i} c_{1} \omega y} f_{1}(y) \mathrm{d} y\right|^{2}+\left|\int_{-1}^{0} e^{-\mathrm{i} c_{2} \omega y} f_{2}(y) \mathrm{d} y\right|^{2} .
\end{aligned}
$$


Proof. It follows from (1.3) that we have

$$
\begin{aligned}
\omega u(-1, \omega)= & \int_{0}^{1} \frac{\mathrm{i}}{c_{1}+c_{2}} e^{\mathrm{i}\left(c_{1} \omega y+c_{2} \omega\right)} f_{1}(y) \mathrm{d} y+\int_{-1}^{0} \frac{\mathrm{i}\left(c_{2}-c_{1}\right)}{2 c_{2}\left(c_{1}+c_{2}\right)} e^{-\mathrm{i} c_{2} \omega(-1+y)} f_{2}(y) \mathrm{d} y \\
& +\int_{-1}^{0} \frac{\mathrm{i}}{2 c_{2}} e^{-\mathrm{i} c_{2} \omega(-1-y)} f_{2}(y) \mathrm{d} y
\end{aligned}
$$

and

$$
\begin{aligned}
\omega u(1, \omega)= & \int_{0}^{1} \frac{\mathrm{i}\left(c_{1}-c_{2}\right)}{2 c_{1}\left(c_{1}+c_{2}\right)} e^{\mathrm{i} c_{1} \omega(1+y)} f_{1}(y) \mathrm{d} y+\int_{0}^{1} \frac{\mathrm{i}}{2 c_{1}} e^{\mathrm{i} c_{1} \omega(1-y)} f_{1}(y) \mathrm{d} y \\
& +\int_{-1}^{0} \frac{\mathrm{i}}{c_{1}+c_{2}} e^{\mathrm{i}\left(-c_{2} \omega y+c_{1} \omega\right)} f_{2}(y) \mathrm{d} y .
\end{aligned}
$$

The proof is done by taking square of the amplitudes on both sides of the above equations.

Next, let

where

$$
I(s)=I_{1}(s)+I_{2}(s)
$$

$$
I_{1}(s)=\omega^{2} \int_{0}^{s}|u(-1, \omega)|^{2} \mathrm{~d} \omega, \quad I_{2}(s)=\omega^{2} \int_{0}^{s}|u(1, \omega)|^{2} \mathrm{~d} \omega .
$$

We have the following explicit representations for $I_{1}(s)$ and $I_{2}(s)$ :

$$
\begin{aligned}
I_{1}(s)= & \int_{0}^{s} \mid \int_{0}^{1} \frac{1}{c_{1}+c_{2}} e^{\mathrm{i}\left(c_{1} \omega y+c_{2} \omega\right)} f_{1}(y) \mathrm{d} y+\int_{-1}^{0} \frac{c_{2}-c_{1}}{2 c_{2}\left(c_{1}+c_{2}\right)} e^{-\mathrm{i} c_{2} \omega(-1+y)} f_{2}(y) \mathrm{d} y \\
& +\left.\int_{-1}^{0} \frac{1}{2 c_{2}} e^{-\mathrm{i} c_{2} \omega(-1-y)} f_{2}(y) \mathrm{d} y\right|^{2} \mathrm{~d} \omega
\end{aligned}
$$

and

$$
\begin{aligned}
I_{2}(s)= & \int_{0}^{s} \mid \int_{0}^{1} \frac{c_{1}-c_{2}}{2 c_{1}\left(c_{1}+c_{2}\right)} e^{\mathrm{i} c_{1} \omega(1+y)} f_{1}(y) \mathrm{d} y+\int_{0}^{1} \frac{1}{2 c_{1}} e^{\mathrm{i} c_{1} \omega(1-y)} f_{1}(y) \mathrm{d} y \\
& +\left.\int_{-1}^{0} \frac{1}{c_{1}+c_{2}} e^{\mathrm{i}\left(-c_{2} \omega y+c_{1} \omega\right)} f_{2}(y) \mathrm{d} y\right|^{2} \mathrm{~d} \omega .
\end{aligned}
$$

Lemma 3.3. Let $f \in L^{2}(-1,1)$ and $c_{\max }=\max \left\{c_{1}, c_{2}\right\}$. We have for any $s=s_{1}+\mathrm{i} s_{2}, s_{1}, s_{2} \in \mathbb{R}$ that

$$
\begin{aligned}
& \left|I_{1}(s)\right| \lesssim|s| e^{4 c_{\max }\left|s_{2}\right|} \int_{0}^{1}|f(y)|^{2} \mathrm{~d} y \\
& \left|I_{2}(s)\right| \lesssim|s| e^{4 c_{\max }\left|s_{2}\right|} \int_{0}^{1}|f(y)|^{2} \mathrm{~d} y .
\end{aligned}
$$

Proof. Let $\omega=s t, t \in(0,1)$. A simple calculation yields

$$
\begin{aligned}
I_{1}(s)= & s \int_{0}^{1} \mid \int_{0}^{1} \frac{1}{c_{1}+c_{2}} e^{\mathrm{i}\left(c_{1} s t y+c_{2} s t\right)} f_{1}(y) \mathrm{d} y+\int_{-1}^{0} \frac{c_{2}-c_{1}}{2 c_{2}\left(c_{1}+c_{2}\right)} e^{-\mathrm{i} c_{2} s t(-1+y)} f_{2}(y) \mathrm{d} y \\
& +\left.\int_{-1}^{0} \frac{1}{2 c_{2}} e^{-\mathrm{i} c_{2} s t(-1-y)} f_{2}(y) \mathrm{d} y\right|^{2} \mathrm{~d} t
\end{aligned}
$$

and

$$
\begin{aligned}
I_{2}(s)= & s \int_{0}^{1} \mid \int_{0}^{1} \frac{c_{1}-c_{2}}{2 c_{1}\left(c_{1}+c_{2}\right)} e^{\mathrm{i} c_{1} s t(1+y)} f_{1}(y) \mathrm{d} y+\int_{0}^{1} \frac{1}{2 c_{1}} e^{\mathrm{i} c_{1} s t(1-y)} f_{1}(y) \mathrm{d} y \\
& +\left.\int_{-1}^{0} \frac{1}{c_{1}+c_{2}} e^{\mathrm{i}\left(-c_{2} s t y+c_{1} s t\right)} f_{2}(y) \mathrm{d} y\right|^{2} \mathrm{~d} t .
\end{aligned}
$$


Noting

$$
\left|e^{ \pm \mathrm{i}\left(c_{1} s t y+c_{2} s t\right)}\right| \leq e^{2 c_{\max }\left|s_{2}\right|}, \quad\left|e^{ \pm \mathrm{i} c_{2} s t(-1 \pm y)}\right| \leq e^{2 c_{\max }\left|s_{2}\right|}, \quad \forall y \in(-1,1),
$$

we have from the Schwartz inequality that

$$
\left|I_{1}(s)\right| \lesssim|s| e^{4 c_{\max }\left|s_{2}\right|} \int_{-1}^{1}|f(y)|^{2} \mathrm{~d} y
$$

Similarly noting

$$
\left|e^{ \pm \mathrm{i} c_{1} s t(1 \pm y)}\right| \leq e^{2 c_{\max }\left|s_{2}\right|}, \quad\left|e^{ \pm \mathrm{i}\left(-c_{2} s t y+c_{1} s t\right)}\right| \leq e^{2 c_{\max }\left|s_{2}\right|}, \quad \forall y \in(-1,1),
$$

we get from the Schwartz inequality that

$$
\left|I_{2}(s)\right| \lesssim|s| e^{4 c_{\max }\left|s_{2}\right|} \int_{-1}^{1}|f(y)|^{2} \mathrm{~d} y
$$

which completes the proof.

Lemma 3.4. Let $f \in H^{n}(-1,1), \operatorname{supp} f \subset(-1,1)$. We have for any $s>0$ that

$$
\int_{s}^{\infty} \omega^{2}\left(|u(-1, \omega)|^{2}+|u(1, \omega)|^{2}\right) \mathrm{d} \omega \lesssim s^{-(2 n-1)}\|f\|_{H^{n}(-1,1)}^{2}
$$

Proof. It follows from Lemma 3.2 that we have

$$
\begin{aligned}
& \int_{s}^{\infty} \omega^{2}|u(-1, \omega)|^{2} \mathrm{~d} \omega+\int_{s}^{\infty} \omega^{2}|u(1, \omega)|^{2} \mathrm{~d} \omega \\
\lesssim & \int_{s}^{\infty}\left|\int_{0}^{1} e^{\mathrm{i} c_{1} \omega y} f_{1}(y) \mathrm{d} y\right|^{2} \mathrm{~d} \omega+\int_{s}^{\infty}\left|\int_{0}^{1} e^{-\mathrm{i} c_{1} \omega y} f_{1}(y) \mathrm{d} y\right|^{2} \mathrm{~d} \omega \\
& +\int_{s}^{\infty}\left|\int_{-1}^{0} e^{\mathrm{i} c_{2} \omega y} f_{2}(y) \mathrm{d} y\right|^{2} \mathrm{~d} \omega+\int_{s}^{\infty}\left|\int_{-1}^{0} e^{-\mathrm{i} c_{2} \omega y} f_{2}(y) \mathrm{d} y\right|^{2} \mathrm{~d} \omega .
\end{aligned}
$$

Using the integration by parts and noting $\operatorname{supp} f_{1} \subset(0,1)$ and $\operatorname{supp} f_{2} \subset(-1,0)$, we obtain

$$
\int_{0}^{1} e^{ \pm \mathrm{i} c_{1} \omega y} f_{1}(y) \mathrm{d} y=\frac{1}{\left( \pm \mathrm{i} c_{1} \omega\right)^{n}} \int_{0}^{1} e^{ \pm \mathrm{i} c_{1} \omega y} f_{1}^{(n)}(y) \mathrm{d} y
$$

and

which give

$$
\int_{-1}^{0} e^{ \pm \mathrm{i} c_{2} \omega y} f_{2}(y) \mathrm{d} y=\frac{1}{\left( \pm \mathrm{i} c_{2} \omega\right)^{n}} \int_{-1}^{0} e^{ \pm \mathrm{i} c_{2} \omega y} f_{2}^{(n)}(y) \mathrm{d} y
$$

and

$$
\left|\int_{0}^{1} e^{ \pm \mathrm{i} c_{1} \omega y} f_{1}(y) \mathrm{d} y\right|^{2} \lesssim c_{1}^{-2 n} \omega^{-2 n}\left\|f_{1}\right\|_{H^{n}(0,1)}^{2} \lesssim c_{1}^{-2 n} \omega^{-2 n}\|f\|_{H^{n}(-1,1)}^{2}
$$

$$
\left|\int_{-1}^{0} e^{ \pm \mathrm{i} c_{2} \omega y} f_{2}(y) \mathrm{d} y\right|^{2} \lesssim c_{2}^{-2 n} \omega^{-2 n}\left\|f_{2}\right\|_{H^{n}(-1,0)}^{2} \lesssim c_{2}^{-2 n} \omega^{-2 n}\|f\|_{H^{n}(-1,1)}^{2} .
$$

Hence we have

$$
\int_{s}^{\infty}\left|\int_{0}^{1} e^{ \pm \mathrm{i} c_{1} \omega y} f_{1}(y) \mathrm{d} y\right|^{2} \mathrm{~d} \omega \lesssim c_{1}^{-2 n}\left\|f_{1}\right\|_{H^{n}(0,1)}^{2} \int_{s}^{\infty} \omega^{-2 n} \mathrm{~d} \omega \lesssim c_{1}^{-2 n} \frac{s^{-(2 n-1)}}{(2 n-1)}\|f\|_{H^{n}(-1,1)}^{2}
$$

and

$$
\int_{s}^{\infty}\left|\int_{-1}^{0} e^{ \pm \mathrm{i} c_{2} \omega y} f_{2}(y) \mathrm{d} y\right|^{2} \mathrm{~d} \omega \lesssim c_{2}^{-2 n}\left\|f_{2}\right\|_{H^{n}(-1,0)}^{2} \int_{s}^{\infty} \omega^{-2 n} \mathrm{~d} \omega \lesssim c_{2}^{-2 n} \frac{s^{-(2 n-1)}}{(2 n-1)}\|f\|_{H^{n}(-1,1)}^{2}
$$

which completes the proof.

The following lemma is proved in [8]. 
Lemma 3.5. Denote $S=\left\{z=x+\mathrm{i} y \in \mathbb{C}:-\frac{\pi}{4}<\arg z<\frac{\pi}{4}\right\}$. Let $J(z)$ be analytic in $S$ and continuous in $\bar{S}$ satisfying

$$
\left\{\begin{array}{l}
|J(z)| \leq \epsilon, \quad z \in(0, L] \\
|J(z)| \leq V, \quad z \in S \\
|J(0)|=0
\end{array}\right.
$$

Then there exits a function $\mu(z)$ satisfying

$$
\begin{cases}\mu(z) \geq \frac{1}{2}, & z \in\left(L, 2^{\frac{1}{4}} L\right), \\ \mu(z) \geq \frac{1}{\pi}\left(\left(\frac{z}{L}\right)^{4}-1\right)^{-\frac{1}{2}}, & z \in\left(2^{\frac{1}{4}} L, \infty\right)\end{cases}
$$

such that

$$
|J(z)| \leq V \epsilon^{\mu(z)}, \quad \forall z \in(L, \infty) .
$$

Lemma 3.6. Let $f \in \mathcal{F}_{M}$. Then there exists a function $\mu(s)$ satisfying

$$
\begin{cases}\mu(s) \geq \frac{1}{2}, & s \in\left(K, 2^{\frac{1}{4}} K\right), \\ \mu(s) \geq \frac{1}{\pi}\left(\left(\frac{s}{K}\right)^{4}-1\right)^{-\frac{1}{2}}, & s \in\left(2^{\frac{1}{4}} K, \infty\right),\end{cases}
$$

such that

$$
|I(s)| \lesssim M^{2} e^{a s} \epsilon^{2 \mu(s)}, \quad \forall s \in(K, \infty)
$$

where $a=\max \{5 c, 3\}$.

Proof. It follows from Lemma 3.3 that

$$
\left|I_{1}(s) e^{-a s}\right| \lesssim M^{2}, \quad\left|I_{2}(s) e^{-a s}\right| \lesssim M^{2}, \quad s \in S .
$$

Recalling (2.2), (3.18), and (3.19), we have

$$
\left|I_{1}(s) e^{-a s}\right| \lesssim \epsilon^{2}, \quad\left|I_{2}(s) e^{-a s}\right| \lesssim \epsilon^{2}, \quad s \in[0, K]
$$

A direct application of Lemma 3.5 shows that there exists a function $\mu(s)$ satisfying (3.20) such that

$$
\left|I_{1}(s) e^{-a s}\right| \lesssim M^{2} \epsilon^{2 \mu}, \quad\left|I_{2}(s) e^{-a s}\right| \lesssim M^{2} \epsilon^{2 \mu}, \quad s \in(K, \infty)
$$

then we have

$$
\left|I(s) e^{-a s}\right|=\left|I_{1}(s) e^{-a s}+I_{2}(s) e^{-a s}\right| \lesssim M^{2} \epsilon^{2 \mu}, \quad s \in(K, \infty),
$$

which completes the proof.

Now we show the proof of Theorem 2.1,

Proof. We can assume that $\epsilon<e^{-1}$, otherwise the estimate is obvious. Let

$$
s= \begin{cases}\frac{1}{(3 \pi)^{\frac{1}{3}}} K^{\frac{2}{3}}|\ln \epsilon|^{\frac{1}{4}}, & 2^{\frac{1}{4}}(3 \pi)^{\frac{1}{3}} K^{\frac{1}{3}}<|\ln \epsilon|^{\frac{1}{4}}, \\ K, & |\ln \epsilon| \leq 2^{\frac{1}{4}}(3 \pi)^{\frac{1}{3}} K^{\frac{1}{3}} .\end{cases}
$$

If $2^{\frac{1}{4}}(3 \pi)^{\frac{1}{3}} K^{\frac{1}{3}}<|\ln \epsilon|^{\frac{1}{4}}$, then we have

$$
\begin{aligned}
|I(s)| & \lesssim M^{2} e^{a s} e^{-\frac{2|\ln \epsilon|}{\pi}\left(\left(\frac{s}{K}\right)^{4}-1\right)^{-\frac{1}{2}}} \lesssim M^{2} e^{\frac{a}{(3 \pi)^{\frac{1}{3}}} K^{\frac{2}{3}}|\ln \epsilon|^{\frac{1}{4}}-\frac{2|\ln \epsilon|}{\pi}\left(\frac{K}{s}\right)^{2}} \\
& =M^{2} e^{-2\left(\frac{a^{3}}{3 \pi}\right)^{\frac{1}{3}} K^{\frac{2}{3}}|\ln \epsilon|^{\frac{1}{2}}\left(1-\frac{1}{2}|\ln \epsilon|^{-\frac{1}{4}}\right)} .
\end{aligned}
$$

Noting $\frac{1}{2}|\ln \epsilon|^{-\frac{1}{4}}<\frac{1}{2}$ and $a \geq 3$, we have $\left(\frac{a^{3}}{3 \pi}\right)^{\frac{1}{3}} \geq\left(\frac{3^{3}}{3 \pi}\right)^{\frac{1}{3}}>1$ and

$$
|I(s)| \lesssim M^{2} e^{-K^{\frac{2}{3}}|\ln \epsilon|^{\frac{1}{2}}}
$$


Using the elementary inequality

we get

$$
e^{-x} \leq \frac{(6 n-3) !}{x^{3(2 n-1)}}, \quad x>0
$$

$$
|I(s)| \lesssim \frac{M^{2}}{\left(\frac{K^{2} \mid \ln \epsilon \epsilon^{\frac{3}{2}}}{(6 n-3)^{3}}\right)^{2 n-1}} .
$$

If $|\ln \epsilon| \leq 2^{\frac{1}{4}}(3 \pi)^{\frac{1}{3}} K^{\frac{1}{3}}$, then $s=K$. We have from (2.2) and Lemma 3.2 that

$$
|I(s)| \leq \epsilon^{2} .
$$

Hence we obtain from Lemma 3.4 that

$$
\begin{aligned}
& \int_{0}^{\infty} \omega^{2}\left(|u(-1, \omega)|^{2}+|u(1, \omega)|^{2}\right) \mathrm{d} \omega \\
& \lesssim \epsilon^{2}+\frac{\|f\|_{H^{n}(-1,1)}^{2}}{\left(\frac{K^{2}|\ln \epsilon|^{\frac{3}{2}}}{(6 n-3)^{3}}\right)^{2 n-1}}+\frac{\|{ }^{2 n-1}}{\left(2^{-\frac{1}{4}}(3 \pi)^{-\frac{1}{3}} K^{\frac{2}{3}}|\ln \epsilon|^{\frac{1}{4}}\right)^{2 n-1}}
\end{aligned}
$$

By Lemma 3.1, we have

$$
\|f\|_{L^{2}(-1,1)}^{2} \lesssim \epsilon^{2}+\frac{M^{2}}{\left(\frac{K^{2}|\ln \epsilon|^{\frac{3}{2}}}{(6 n-3)^{3}}\right)^{2 n-1}}+\frac{M^{2}}{\left(\frac{\left.K^{\frac{2}{3}} \ln \epsilon\right|^{\frac{1}{4}}}{(6 n-3)^{3}}\right)^{2 n-1}} .
$$

Since $K^{\frac{2}{3}}|\ln \epsilon|^{\frac{1}{4}} \leq K^{2}|\ln \epsilon|^{\frac{3}{2}}$ when $K>1$ and $|\ln \epsilon|>1$, we obtain the stability estimate.

\section{Conclusion}

In this paper, we show that the increasing stability can be obtained for the inverse source scattering problem of the one-dimensional Helmholtz equation in a two-layered medium by using multifrequency Dirichlet data at the two end points of an interval which contains the compact support of the source. The stability estimate consists of the data discrepancy and the high frequency tail of the source function. We believe that the proposed method can be extended to handle a multi-layered medium. Another possible future work is to investigate the higher dimensional problem.

\section{Appendix A. Green's function in a two-layered medium}

Consider the equation

$$
\frac{\mathrm{d}^{2} g(x, y)}{\mathrm{d} x^{2}}+\kappa^{2}(x) g(x, y)=-\delta(x-y)
$$

where $\delta$ is the Dirac delta function and the wavenumber $\kappa$ is a piecewise constant, i.e.,

$$
\kappa(x)= \begin{cases}\kappa_{1}, & x>0 \\ \kappa_{2}, & x<0\end{cases}
$$

If $y>0$, the solution of (A.1) has the following form

$$
g(x, y)= \begin{cases}A e^{\mathrm{i} \kappa_{1} x}, & x>y, \\ B e^{\mathrm{i} \kappa_{1} x}+C e^{-\mathrm{i} \kappa_{1} x}, & 0<x<y, \\ D e^{-\mathrm{i} \kappa_{2} x}, & x<0,\end{cases}
$$


where $A, B, C, D$ are to be determined. Using the continuity conditions

$$
\left\{\begin{array}{l}
\left.g(x, y)\right|_{x=y^{+}}=\left.g(x, y)\right|_{x=y^{-}}, \\
\left.\frac{\mathrm{d} g(x, y)}{\mathrm{d} x}\right|_{x=y^{+}}-\left.\frac{\mathrm{d} g(x, y)}{\mathrm{d} x}\right|_{x=y^{-}}=-1, \\
\left.g(x, y)\right|_{x=0^{+}}=\left.g(x, y)\right|_{x=0^{-}}, \\
\left.\frac{\mathrm{d} g(x, y)}{\mathrm{d} x}\right|_{x=0^{+}}=\left.\frac{\mathrm{d} g(x, y)}{\mathrm{d} x}\right|_{x=0^{-}},
\end{array}\right.
$$

we get a linear system:

$$
\left\{\begin{array}{l}
A e^{\mathrm{i} \kappa_{1} y}=B e^{\mathrm{i} \kappa_{1} y}+C e^{-\mathrm{i} \kappa_{1} y}, \\
\mathrm{i} \kappa_{1} A e^{\mathrm{i} \kappa_{1} y}-\mathrm{i} \kappa_{1} B e^{\mathrm{i} \kappa_{1} y}+\mathrm{i} \kappa_{1} C e^{-\mathrm{i} \kappa_{1} y}=-1, \\
B+C=D, \\
\mathrm{i} \kappa_{1} B-\mathrm{i} \kappa_{1} C=-\mathrm{i} \kappa_{2} D .
\end{array}\right.
$$

A simple calculation yields that

$$
\left\{\begin{array}{l}
A=\mathrm{i} \frac{\kappa_{1}-\kappa_{2}}{2 \kappa_{1}\left(\kappa_{1}+\kappa_{2}\right)} e^{\mathrm{i} \kappa_{1} y}+\frac{\mathrm{i}}{2 \kappa_{1}} e^{-\mathrm{i} \kappa_{1} y} \\
B=\mathrm{i} \frac{\kappa_{1}-\kappa_{2}}{2 \kappa_{1}\left(\kappa_{1}+\kappa_{2}\right)} e^{\mathrm{i} \kappa_{1} y} \\
C=\frac{\mathrm{i}}{2 \kappa_{1}} e^{\mathrm{i} \kappa_{1} y} \\
D=\frac{\mathrm{i}}{\kappa_{1}+\kappa_{2}} e^{\mathrm{i} \kappa_{1} y}
\end{array}\right.
$$

which gives

$$
g(x, y)= \begin{cases}\mathrm{i} \frac{\kappa_{1}-\kappa_{2}}{2 \kappa_{1}\left(\kappa_{1}+\kappa_{2}\right)} e^{\mathrm{i} \kappa_{1}(x+y)}+\frac{\mathrm{i}}{2 \kappa_{1}} e^{\mathrm{i} \kappa_{1}|x-y|}, & x>0, \\ \frac{\mathrm{i}}{\kappa_{1}+\kappa_{2}} e^{\mathrm{i}\left(\kappa_{1} y-\kappa_{2} x\right)}, & x<0 .\end{cases}
$$

If $y<0$, the solution has the following form

$$
g(x, y)= \begin{cases}A e^{-\mathrm{i} \kappa_{2} x}, & x<y, \\ B e^{-\mathrm{i} \kappa_{2} x}+C e^{\mathrm{i} \kappa_{2} x}, & y<x<0, \\ D e^{\mathrm{i} \kappa_{1} x}, & x>0 .\end{cases}
$$

Using the continuity conditions

$$
\left\{\begin{array}{l}
\left.g(x, y)\right|_{x=y^{+}}=\left.g(x, y)\right|_{x=y^{-}}, \\
\left.\frac{\mathrm{d} g(x, y)}{\mathrm{d} x}\right|_{x=y^{+}}-\left.\frac{\mathrm{d} g(x, y)}{\mathrm{d} x}\right|_{x=y^{-}}=-1, \\
\left.g(x, y)\right|_{x=0^{+}}=\left.g(x, y)\right|_{x=0^{-}}, \\
\left.\frac{\mathrm{d} g(x, y)}{\mathrm{d} x}\right|_{x=0^{+}}=\left.\frac{\mathrm{d} g(x, y)}{\mathrm{d} x}\right|_{x=0^{-}},
\end{array}\right.
$$

we obtain

$$
\left\{\begin{array}{l}
A e^{-\mathrm{i} \kappa_{2} y}=B e^{-\mathrm{i} \kappa_{2} y}+C e^{\mathrm{i} \kappa_{2} y} \\
-\mathrm{i} \kappa_{2} A e^{-\mathrm{i} \kappa_{2} y}+\mathrm{i} \kappa_{2} B e^{-\mathrm{i} \kappa_{2} y}-\mathrm{i} \kappa_{2} C e^{\mathrm{i} \kappa_{2} y}=1 \\
B+C=D \\
-\mathrm{i} \kappa_{2} B+\mathrm{i} \kappa_{2} C=\mathrm{i} \kappa_{1} D
\end{array}\right.
$$

It follows from solving the above linear system that

$$
\left\{\begin{array}{l}
A=\mathrm{i} \frac{\kappa_{2}-\kappa_{1}}{2 \kappa_{2}\left(\kappa_{1}+\kappa_{2}\right)} e^{-\mathrm{i} \kappa_{2} y}+\frac{\mathrm{i}}{2 \kappa_{2}} e^{\mathrm{i} \kappa_{2} y} \\
B=\mathrm{i} \frac{\kappa_{2}-\kappa_{1}}{2 \kappa_{2}\left(\kappa_{1}+\kappa_{2}\right)} e^{-\mathrm{i} \kappa_{2} y} \\
C=\frac{\mathrm{i}}{2 \kappa_{2}} e^{-\mathrm{i} \kappa_{2} y} \\
D=\frac{\mathrm{i}}{\kappa_{1}+\kappa_{2}} e^{-\mathrm{i} \kappa_{2} y}
\end{array}\right.
$$


which yields

$$
g(x, y)= \begin{cases}\mathrm{i} \frac{\kappa_{2}-\kappa_{1}}{2 \kappa_{2}\left(\kappa_{1}+\kappa_{2}\right)} e^{-\mathrm{i} \kappa_{2}(x+y)}+\frac{\mathrm{i}}{2 \kappa_{2}} e^{\mathrm{i} \kappa_{2}|x-y|}, & x<0, \\ \frac{\mathrm{i}}{\kappa_{1}+\kappa_{2}} e^{\mathrm{i}\left(-\kappa_{2} y+\kappa_{1} x\right)}, & x>0 .\end{cases}
$$

\section{REFERENCES}

[1] S. Acosta, S. Chow , J. Taylor, and V. Villamizar, On the multi-frequency inverse source problem in heterogeneous media, Inverse Problems, 28 (2012), 075013.

[2] R. Albanese and P. Monk, The inverse source problem for Maxwell's equations, Inverse Problems, 22 (2006), 1023-1035.

[3] H. Ammari, G. Bao, and J. Fleming, An inverse source problem for Maxwell's equations in magnetoencephalography, SIAM J. Appl. Math., 62 (2002), 1369-1382.

[4] S. Arridge, Optical tomography in medical imaging, Inverse Problems, 15 (1999), R41R93.

[5] A. Badia and T. Nara, An inverse source problem for Helmholtz's equation from the Cauchy data with a single wave number, Inverse Problems, 27 (2011), 105001.

[6] G. Bao, P. Li, J. Lin, and F. Triki, Inverse scattering problems with multi-frequencies, Inverse Problems, 31 (2015), 093001.

[7] G. Bao, J. Lin, and F. Triki, A multi-frequency inverse source problem, J. Differential equations, 249 (2010) 3443-3465.

[8] J. Cheng, V. Isakov, and S. Lu, Increasing stability in the inverse source problem with many frequencies, J. Differential Equations, 260 (2016), 4786-4804.

[9] A. Devaney, E. Marengo, and M. Li, Inverse source problem in nonhomogeneous background media, SIAM J. Appl. Math., 67 (2007), 1353-1378.

[10] A. Devaney and G. Sherman, Nonuniqueness in inverse source and scattering problems, IEEE Trans. Antennas Propag., 30 (1982), 1034-1037.

[11] M. Eller and N. Valdivia, Acoustic source identification using multiple frequency information, Inverse Problems, 25 (2009), 115005.

[12] K.-H. Hauer, L. Kühn, and R. Potthast, On uniqueness and non-uniqueness for current reconstruction from magnetic fields, Inverse Problems, 21 (2005), 955-967.

[13] V. Isakov, Inverse Source Problems, AMS, Providence, RI, 1989.

[14] P. Li and G. Yuan, Increasing stability for the inverse source scattering problem with multi-frequencies, Inverse Problems and Imaging, 11 (2017), 745-759.

[15] P. Li and G. Yuan, Stability on the inverse random source scattering problem for the one-dimensional Helmholtz equation, J. Math. Anal. Appl., 450 (2017), 872-887.

School of Mathematics and Statistics, Jiangsu Normal University, Xuzhou, Jiangsu 221116 , China. E-mail address: zhaoy@jsnu.edu.cn

Department of Mathematics, Purdue University, West Lafayette, Indiana 47907, USA.

E-mail address: lipeijun@math.purdue.edu 